\title{
Quasicrystal-Enhanced Nucleation During the Solidification of fcc Metallic Alloys: A Tentative Thermodynamic Approach
}

\author{
Michel Rappaz and Güven Kurtuldu
}

\begin{abstract}
This short contribution is an extended abstract which summarizes a recent discovery we have made regarding the nucleation of fcc grains during the solidification of metallic alloys. While trying to analyze the effects of minute solute additions to the directional solidification of aluminum alloys, we observe that $200 \mathrm{ppm}$ of $\mathrm{Cr}$ added to Al-
\end{abstract} 20 wt. $\%$ Zn alloys induced a change of the primary trunk growth direction from $\langle 100\rangle$ to $\langle 110\rangle .^{[1]}$ Such a change of growth directions, so-called dendrite orientation transition (DOT), has been evidenced in the Al-Zn system when the zinc composition is increased from about 25 to 55 wt. $\%{ }^{[2-4]}$ It has been attributed to a change of the solid-liquid interfacial energy anisotropy, since zinc is a highly anisotropic element which has a very high solubility in aluminum at high temperature. ${ }^{[5]}$ In the present case, it seems that a few hundreds ppm of $\mathrm{Cr}$ produce the same effect for $\mathrm{Al}-\mathrm{Zn}$ alloys having a composition at the lower limit of the DOT.

Investigating then the behavior of Al-20 wt.\%Zn-Cr alloys under equiaxed solidification conditions, we found out that chromium had a drastic effect on the final grain size for otherwise identical cooling conditions. ${ }^{[1,6]}$ Besides grain refinement, detailed EBSD analyses showed that: (i) chromium induces an abnormal percentage of twinned grain boundaries (more than 2\%); (ii) some of these grain boundaries are in a neartwin relationship, i.e., a twin relationship with a rotation of $5^{\circ}-7^{\circ}$ around a common $\langle 110\rangle$ direction; (iii) several nearest-neighbor (nn) grains exhibit multiple twin or near-twin relationships with a common $\langle 110\rangle$ direction. These observations are a strong indi- cation that a template must induce the nucleation of the fcc phase.

Considering the phase diagram of Al-Zn-Cr, ${ }^{[7]}$ Al-20 wt.\%Zn-0.1 wt.\% $\mathrm{Cr}$ has the liquidus of the intermetallic phase $\mathrm{Al}_{13} \mathrm{Cr}_{2}$ above that of the fcc phase, i.e., this phase can form first during solidification. Furthermore, $\mathrm{Al}_{13} \mathrm{Cr}_{2}$ (also identified as $\mathrm{Al}_{7} \mathrm{Cr}$ or $\mathrm{Al}_{45} \mathrm{Cr}_{7}$ ) contains many icosahedral building blocks in its large monoclinic unit cell, ${ }^{[8]}$ with $\mathrm{Cr}$ atoms at the center of each of them. It is the approximant phase of $\mathrm{Al}_{7} \mathrm{Cr}$ icosahedral quasicrystals (iQC). iQC's have a very low interfacial energy with the liquid, ${ }^{[9]}$ mainly because their structure is very close to that of the liquid, for which atomic icosahedral short-range order (ISRO) was postulated by Frank already in the 1950s. ${ }^{[10]}$ Since the nucleation barrier is a cubic power of the interfacial energy, the undercooling required for the formation of iQC's is much lower than that of the fcc phase (see Fig. 1). ${ }^{[1]}$

Based on the observations made on the Al- $\mathrm{Zn}-\mathrm{Cr}$ system and on theoretical considerations, we then proposed the following nucleation mechanism (see Fig. 2): (i) iQC's form in the liquid from ISRO of atoms in the liquid; (ii) They grow, thus depleting the melt in $\mathrm{Cr}$ atoms (the system is peritectic $\left.^{[12]}\right)$. At some point the fcc phase forms heteroepitaxially on the iQC's facets with the following relationships: $\langle 111\rangle_{\mathrm{fcc}} / /$ threefold symmetry axes of the iQC, and $\langle 110\rangle_{\mathrm{fcc}} \perp$ twofold symmetry axes of the iQC. This induces multiply-twinned fcc grains, which share a common $\langle 110\rangle$ direction when they are issued from five facets of an iQC sharing a fivefold symmetry axis; (iii) Upon further cooling, the iQC template can even disappear by a peritectic transformation.

Similar observations, with an even more drastic effect, have then been made on yellow gold (Au-12.5wt. $\% \mathrm{Ag}-12.5 \mathrm{wt} . \% \mathrm{Cu}$ ) in which 20200 ppm of iridium are added. ${ }^{[13]}$ In this case, the grain size can be decreased by a factor 10 (a factor $10^{3}$ in the grain density) with the addition of 200 ppm Ir. Nine multiply-twinned fcc nn grains could be found in this case which reproduced with their common $\langle 110\rangle$ directions the six fivefold symmetry axes of an iQC.

In a recent contribution presented at the occasion of this Hume-Rothery symposium, ${ }^{[11]}$ we have suggested, based on thermodynamic considerations, that the iQC-mediated nucleation of the fcc phase could be viewed in a way similar to precipitation of a stable second phase from a supersaturated solid solution (e.g., a sequence of metastable phases such as $\theta " \rightarrow$ $\theta^{\prime} \rightarrow \theta \mathrm{Al}_{2} \mathrm{Cu}$ in $\mathrm{Al}-\mathrm{Cu}$ or $\alpha^{\prime}{ }_{\text {rhomb }}$ $\rightarrow \alpha^{\prime} \rightarrow \mathrm{Zn}$ in $\mathrm{Al}-\mathrm{Zn}$ alloys). In such phase transformations, the sequence usually starts from soluterich Guinier-Preston (GP) zones which can be viewed as a spinodal decomposition of the parent phase. In the present case, the formation of iQC's from the liquid could occur by a similar mechanism, i.e., demixing of the liquid leading to iSRO-rich regions which then transform into iQC's. Homogeneous nucleation of iQC's would no longer be considered as a discontinuous process but rather as local fluctuations of icosahedral configurations of atoms in the liquid until a critical size is reached. Once the temperature has decreased and the iQC's have grown, thermodynamic considerations seem to indicate that this is rather a peritectic 


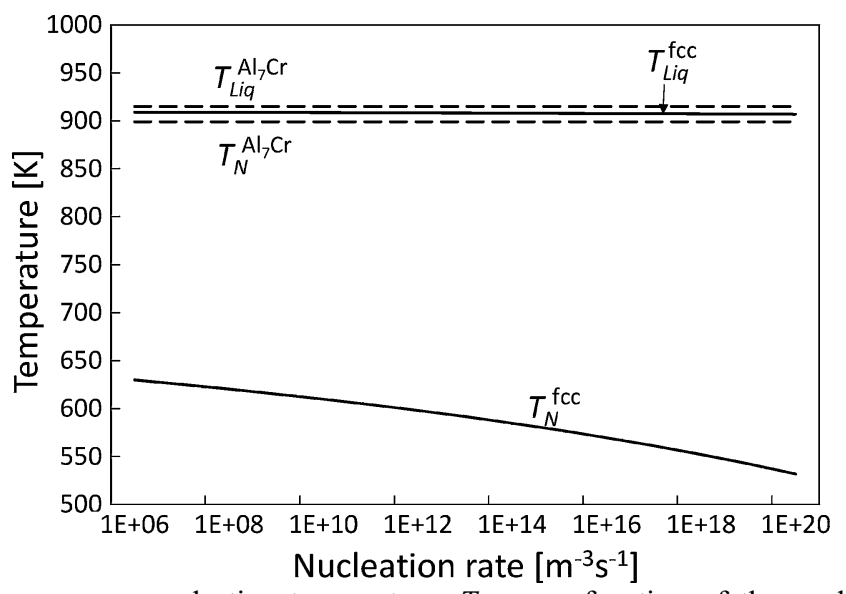

Fig. 1 Homogeneous nucleation temperature, $T_{\mathrm{N}}$, as a function of the nucleation rate for $\mathrm{Al}-20 \mathrm{wt} . \% \mathrm{Zn}$ and for $\mathrm{Al}_{7} \mathrm{Cr}$ iQC's in the same alloy. The thermodynamic data of $\mathrm{Al}_{7} \mathrm{Cr}$ iQC's are taken from the approximant phase, while assuming an interfacial energy $\gamma_{\mathrm{QC}, \ell} \cong 0.1 \gamma_{\mathrm{fcc}, \ell}$ and the pre-exponential factor $I_{0}^{\mathrm{iQC}}=5 \times 10^{-4} \times I_{0}^{\mathrm{fcc}[11]}$

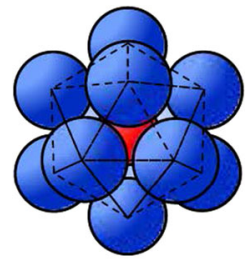

(a) $\AA$

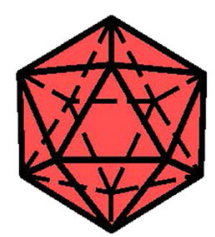

(b) $1-10 \mathrm{~nm}$

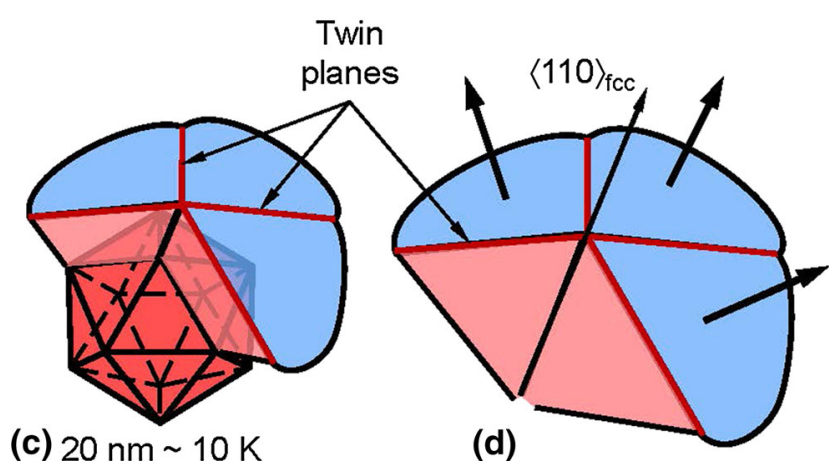

Fig. 2 Schematic diagram showing the iQC-mediated nucleation of the fcc phase during solidification: (a) ISRO atomic arrangement in the liquid; (b) Formation of iQC; (c) Formation of the fcc on the iQC with heteroepitaxy relationship; (d) Further growth of the fcc with disappearance of iQC during cooling, leaving only multiply-twinned fcc grains with some common $\langle 110\rangle$ directions

transformation of the outer layers of the iQC's into a fcc arrangement that promote the transition to fcc grains. ${ }^{[11]}$

\section{References}

1. G. Kurtuldu, "Influence of Trace Elements on the Nucleation and Solidification Morphologies of fcc
Alloys and Relationship with Icosahedral Quasicrystal Formation," Ph.D. Thesis \#6057, EPFL, Lausanne, Switzerland, 2014

2. F. Gonzales and M. Rappaz, Dendrite Growth Directions in Al-Zn Alloys, Met. Mater Trans. A, 2006, 37A, p 2797

3. J. Friedli, J.L. Fife, P. Di Napoli, and M. Rappaz, Dendritic Growth
Morphologies in Al-Zn Alloys-Part I: X-ray Tomographic Microscopy, Met. Mater. Trans. A, 2013, 44A, p 5522-5531. doi:10.1007/s11661-0131912-7

4. J.A. Dantzig, P. Di Napoli, J. Friedli, and M. Rappaz, Dendritic Growth Morphologies in Al-Zn Alloys-Part II: Phase-Field Computations, Metall. Mater. Trans. A, 2013, 44A, p 55325543. doi:10.1007/s11661-013-19118

5. T. Haxhimali, A. Karma, F. Gonzales, and M. Rappaz, Orientation Selection in Dendritic Evolution, Nat. Mater., 2006, 5, p 660

6. G. Kurtuldu, Ph Jarry, and $\mathrm{M}$. Rappaz, Influence of $\mathrm{Cr}$ on the Nucleation of Primary Al and Formation of Twinned Dendrites in $\mathrm{Al}-\mathrm{Zn}-\mathrm{Cr}$ Alloys: Can Icosahedral Solid Clusters Play a Role?, Acta Mater., 2013, 61, p 7098

7. M. Hubert Protopopescu and H. Hubert, Aluminium-Chromium-Zinc, Ternary Alloys, $V C H, 1991,4$, p 449454

8. M.J. Cooper, The Structure of the Intermetallic Phase $\theta(\mathrm{Cr}-\mathrm{Al})$, Acta Cryst., 1960, 13, p 257

9. K.F. Kelton, Quasicrystals: Structure and Stability, Intern. Mater. Rev., 1993, 38, p 105

10. F.C. Frank, Supercooling of Liquids, Proc. R. Soc. Lond. A, 1952, 215, p 43

11. M. Rappaz and G. Kurtuldu, Thermodynamic Aspects of Homogeneous Nucleation Enhanced by Icosahedral Short Range Order in Liquid FccType Alloys, J. Metals, 2015, doi: 10.1007/s11837-015-1328-5

12. G. Kurtuldu, P. Jessner, and M. Rappaz, Peritectic Reaction on the Al-rich Side of Al-Cr System, J. Alloys Compd., 2015, 621, p 283

13. G. Kurtuldu, A. Sicco, and M. Rappaz, Icosahedral QuasicrystalEnhanced Nucleation of the Fcc Phase in Liquid Gold Alloys, Acta Mater, 2014, 70, p 240

Above remarks by:

Michel Rappaz and Güven Kurtuldu, Institute of Materials, Ecole Polytechnique Fédérale de Lausanne, Station 12, 1015 Lausanne, Switzerland.

Contact e-mail: michel.rappaz@epfl.ch. 